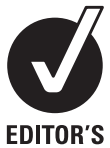

CHOICE
'Department of Neurology, McGill University, Montreal General Hospital, Montreal, Quebec, Canada

${ }^{2}$ Centre d'etude du sommeil, Hopital du Sacre-Coeur.

Montreal, Canada

${ }^{3}$ Département de psychiatrie, Université de Montréal, Canada

\section{Correspondence to} Dr Ronald B Postuma, Department of Neurology, L7-312 Montreal General Hospital, 1650 Cedar Avenue, Montreal, Quebec H3G 1A4, Canada;

ronald.postuma@mcgill.ca

Received 5 February 2009 Revised 18 December 2009

Accepted 23 December 2009 Published Online First

20 June 2010

\title{
Clinical prediction of Parkinson's disease: planning for the age of neuroprotection
}

\author{
R B Postuma, ${ }^{1,2}$ J F Gagnon, ${ }^{2,3}$ J Montplaisir ${ }^{2,3}$
}

\begin{abstract}
As a chronic progressive disease, Parkinson's disease (PD) has a presymptomatic interval; that is, a period during which the pathological process has begun, but motor signs required for the clinical diagnosis are absent. The ability to identify this preclinical stage may be critical in the development and eventual use of neuroprotective therapy. Recently proposed staging systems of PD have suggested that degeneration may occur initially in areas outside the substantia nigra, suggesting that non-motor manifestations may be markers of presymptomatic PD. Decreased olfaction has recently been demonstrated to predict PD in prospective pathological studies, although the lead time may be relatively short, and the positive predictive value is low. Idiopathic RBD has a very high predictive value, with approximately $50 \%$ of affected individuals developing PD or dementia within 10 years. This implies that idiopathic RBD patients are ideal candidates to test potential preclinical markers. However, the specificity of symptom screens for RBD is not established, not all persons with PD develop $\mathrm{RBD}$, and there are only limited ways to predict which RBD patients will develop PD. Other simple screens based upon autonomic symptoms, depression and personality changes, quantitative motor testing and other sleep disorders may also be useful markers, but have not been extensively tested. Other more expensive measures such as detailed autonomic testing, cardiac MIBGscintigraphy, dopaminergic imaging and transcranial ultrasound may be especially useful in defining disease risk in those identified through primary screening.
\end{abstract}

\section{INTRODUCTION}

As a progressive neurodegenerative disorder Parkinson's disease (PD) does not start suddenly; therefore, there is a period during which degeneration is ongoing, but disease is not yet clinically evident. This implies potential to predict PD by detecting this presymptomatic threshold with clinical examination, symptom screens and other markers. This review summarises the goals of PD prediction, the pathophysiological basis for prediction and what is currently known about predictive markers of $\mathrm{PD}$, and discusses how these markers could be implemented in the future, particularly in an age when neuroprotective therapy has been developed.

\section{WHY IS PREDICTION IMPORTANT?}

Development of predictive markers for PD will require a very substantial research investment, and clinical application of predictive methods in the future will be even more costly. Therefore, before beginning to study disease prediction, it is first essential to determine why it is important to do so. Understanding the purpose provides the framework with which potential predictive strategies can be evaluated and compared. One important question is whether foreknowledge of PD is, in itself, a sufficient motivation for using predictive measures. Theoretically, knowledge of an impending neurodegenerative condition could help in planning retirement, finances, family structure, etc. On the other hand, such knowledge could lead to distress, premature adoption of sick role, discrimination or even suicide. An analogous experience of prediction for knowledge's sake is presymptomatic testing for Huntington's disease (HD), for which despite having broad availability, only between $4 \%$ and $24 \%$ of eligible persons elect to have testing. ${ }^{1}$ One important difference, however, is that unlike $\mathrm{HD}, \mathrm{PD}$ manifestations are treated very effectively with medications; surveillance for PD can result in early symptomatic improvement, perhaps with long-term benefits on quality of life.

The most important reason to develop predictive agents becomes apparent upon imagining the future of neurology. The most elusive goal in neurodegeneration is a neuroprotective agent, that is, a therapy to slow or stop the underlying degenerative process. If and when neuroprotective treatment becomes available, it will become essential to identify patients as early as possible. A partially effective agent with minor utility in established disease might slow or even prevent the onset of clinical disease if given in preclinical stages. Therefore, the major use of predictive markers of $\mathrm{PD}$ will be in the future, and present efforts to design predictive markers must plan for an age of neuroprotective therapy.

\section{WHO COULD BE TESTED?}

In an age of neuroprotection, those at high disease risk will be motivated to undergo even relatively invasive procedures to determine need for therapy. However, PD has an approximate prevalence of $1.5 \%$ at age $65,{ }^{2}$ and it is unclear whether high-risk populations will be easily identifiable. Family members of patients with PD could be one potential high-risk population; however, RR for firstdegree relatives is approximately $2-3$, implying a increase in risk to only $4 \% .^{2} 3$ Defined causative gene mutations are identifiable in some patients, and these gene carriers are ideal candidates for studies of predictive markers. However, twin studies have found a low genetic contribution to $\mathrm{PD}$ risk in persons over $50^{3}$ (at least in Caucasian populations), suggesting that the proportion of $\mathrm{PD}$ patients with identifiable causative mutations is 
unlikely to grow substantially. Many environmental risk factors (eg, pesticide use, non-smoking) increase the risk of PD. However, other than age, either ORs for risk factors are low (ie, $\sim 2$ ) or exposures are rare.

If there are no methods to reliably identify persons at substantial risk of disease, screening in an age of neuroprotection may need to be population-wide. This adds considerable additional challenges-in addition to being sensitive and specific, screens must now also be non-invasive and inexpensive. Invasive or expensive tests may eventually be used predominantly as secondary confirmation of a positive screen or primary screens for the few who are definable as high-risk.

\section{PATHOPHYSIOLOGICAL BASIS OF PREDICTOR DEVELOPMENT-PRECLINICAL PD}

The first major principle behind the development of predictive markers is redundancy/compensation. Pathological and neuroimaging studies suggest that motor signs of PD only develop once $50-70 \%$ of SNpc neurons have degenerated. ${ }^{4}$ Since neurodegenerative disorders are progressive, less complete stages of degeneration in the SNpc should be detectible. This is the basis for the use of dopaminergic imaging and quantitative motor testing as disease predictors. Similar redundancy may be present in other non-motor areas (see below), implying that sensitive markers of these systems could predict preclinical disease sooner than clinical symptoms or signs.

Perhaps the most important principle presently guiding predictor development is that PD may not start in the substantia nigra ( $\mathrm{SNpc}$ ). According to a proposed staging system by Braak et al, the first stage of PD involves deposition of $\alpha$ synuclein in the anterior olfactory nucleus and dorsal motor nucleus of the vagus. ${ }^{5}$ Peripheral autonomic ganglia and unmyelinated lamina-1 spinal cord neurons may also be involved in stage $1 .^{6}$ Stage 2 is characterised by medullary and pontine involvement affecting lower raphae, the reticular formation and the coeruleus/subcoeruleus complex. Stage 3 affects midbrain (including SNpc), and at Stages 4-6 cortical structures are affected. With some important modifications and exceptions, investigators in other groups have generally confirmed these findings. ${ }^{7}$ However, some important limitations should be noted. It is unlikely that the same pathological process is followed universally; subsequent studies have found variable progression patterns with onset of disease in the SNpc or multicentrically. ${ }^{7-10}$ This model assessed $\boldsymbol{\alpha}$-synuclein deposition, which may not correlate with neurodegeneration; in some cases, even advanced stages of $\boldsymbol{\alpha}$-synuclein deposition can be present without clinical parkinsonism or dementia. ${ }^{11}$ Clinical histories of patients were incomplete, limiting clinicopathological correlation. Brains were selected for detailed analysis if there was synuclein deposition in the dorsal motor vagus, introducing a potentially important selection bias. Finally, speed of progression through early stages is unknown-rapid progression implies a short premotor interval, limiting the effectiveness of predictive markers. Despite these limitations, the recognition that initial pathology of $\mathrm{PD}$ may occur outside the SNpc suggests that screening for non-motor manifestations may detect earlier stages of PD.

\section{POTENTIAL MARKERS}

For the purposes of this review, a clinical predictive marker for PD will be arbitrarily defined as a sign of insipient neurodegeneration present before PD can be diagnosed clinically. Factors that are generally considered risk factors but not preclinical signs of disease (eg, pesticide use, non-smoking) will not be discussed (note that it is impossible to be sure that some 'risk factors' are not preclinical signs). Ongoing research is identifying CSF and serum markers of disease. ${ }^{12}$ This complex area has, so far, not found reliable predictive biomarkers-for space reasons, this field will not be discussed further.

There are a considerable number of potential markers for prediction of $\mathrm{PD}$. To aid in conceptualisation, the discussion of these markers will be divided according to Braak stages.

\section{'STAGE 1' MARKERS Olfaction}

In Braak Stage $1 \mathrm{PD}$, synuclein deposition predominates in the anterior olfactory nucleus and, to a lesser extent, in the olfactory bulb. ${ }^{13}$ Accordingly, the large majority of PD patients have severe olfactory loss at disease onset. ${ }^{14}$ Olfaction is usually normal or only mildly impaired in other parkinsonian disorders. ${ }^{14}$ Olfactory loss may also be an important preclinical marker of dementia, especially Lewy body dementia (LBD) ${ }^{15}$ and Alzheimer's disease (AD). In general, olfactory loss is less severe in $\mathrm{AD}$ than in $\mathrm{LBD}$, and there have been suggestions that complete anosmia may help identify LBD in a patient with dementia. ${ }^{15}$ A major advantage of olfactory tests is that they are inexpensive and non-invasive. The commonest tests include the University of Pennsylvania Smell Identification Test, ${ }^{16}$ a forcedchoice scratch-and-sniff test and 'Sniffin' Sticks', which are felt pens impregnated with odours. ${ }^{17}$ The latter method tests olfactory threshold as well as discrimination, although the former can be self-administered (even by post) and is more widely used.

There is good evidence that olfactory loss can predict PD. One study measuring olfaction in 400 first-degree relatives of PD patients found that those with impaired olfaction had dopaminergic denervation on $B$-CIT SPECT. ${ }^{18}$ Two years later, 4/40 of hyposmics developed $\mathrm{PD}$, compared with 0/360 of normosmics, ${ }^{19}$ and at 5 years, those who had developed PD had lower mean scores on olfactory tests at baseline. ${ }^{20}$ Another small study of patients with idiopathic hyposmia found that $2 / 24$ developed $\mathrm{PD}$ after 4 years of follow-up. ${ }^{21}$ The strongest evidence for the role of olfaction in PD prediction comes from the Honolulu-Asia Ageing Study. ${ }^{22}$ This pathological study examined olfaction using the Cross-Cultural Smell Identification Test in healthy Asian men, and prospectively correlated this with pathologically confirmed PD. In the first 4 years of follow-up, those in the lowest quartile of olfactory function had an OR of 5.2 for the development of $\mathrm{PD}$, and those in the second lowest quartile had an OR of 3.1 for PD.

Despite this promise, some important caveats must be noted. Lead time (the interval between detection of olfactory abnormality and clinical disease) may be limited. In the Honolulu study, olfactory loss did not predict PD when assessed $>4$ years before disease onset. ${ }^{22}$ Similar findings were found in a smaller twin study of olfaction in $\mathrm{PD}^{23}$ In the study of first-degree relatives, whereas $4 / 40$ hyposmic patients developed $\mathrm{PD}$ in the first 2 years, only one other hyposmic patient had developed PD in the following 3 years. ${ }^{20}$ Specificity may be low-all in the lowest quartile of the Honolulu study had severe hyposmia, but only $10 / 549(2 \%)$ developed PD. ${ }^{22}$ This low specificity suggests that olfactory testing will be insufficient by itself to indicate need for neuroprotective therapy. Finally, olfactory loss will probably also screen persons in preclinical stages of dementia, implying that olfactory testing would be a more effective screen for neuroprotective therapy that has a non-disease-specific action. 


\section{Autonomic dysfunction}

In Stage 1 disease, there is prominent $\alpha$-synuclein staining of unmyelinated projection neurons of the dorsal motor vagus, ${ }^{5}$ and recent studies suggest peripheral postganglionic sympathetic denervation may occur even earlier. ${ }^{62}$ Symptoms of autonomic dysfunction are experienced by $40-70 \%$ of $\mathrm{PD}$ patients, ${ }^{25}$ often at disease onset. There are now two prospective studies suggesting that constipation may predict PD. In the Honolulu study, a single question regarding bowel-movement frequency was asked at baseline. ${ }^{26}$ Those who reported a bowelmovement frequency of $<1 /$ day had a PD OR of 2.3 compared with those with 1 per day, and 4.8 compared with those with $>2$ per day. A second prospective record-linkage study demonstrated a 2.5-fold increased risk of PD in patients with a diagnosis of constipation. ${ }^{27}$ Interestingly, in both studies, this effect was evident $10-20$ years before PD onset-if preclinical stages are shorter than 20 years, constipation may indicate an at-risk state as well as being an early disease manifestation. Other potential simple autonomic measures, such as orthostatic blood pressure, beat-to-beat variability and urinary symptoms, have not been studied as predictors, although abnormalities in beatto-beat variability have been reported in idiopathic RBD (see below). ${ }^{28} 29$

One more expensive measure of autonomic function is cardiac metaiodobenzylguanidine (MIBG) scintigraphy, which measures postganglionic sympathetic cardiac innervation. The majority of patients with PD have abnormal MIBG scintigraphy, which appears to be present at the earliest stages of disease. ${ }^{30}$ MIBG scintigraphy is also abnormal in LBD and may help to distinguish LBD from AD. Recent studies have found abnormal scintigraphy in RBD, another preclinical marker of disease. ${ }^{31}$ Although there has been no direct confirmation of disease prediction, the high sensitivity of MIBG scintigraphy combined with pathological evidence of early sympathetic degeneration suggests considerable potential.

Again, caveats must be noted. First, the direct evidence that autonomic dysfunction predicts disease is less strong than it is for olfaction. Symptoms and signs of autonomic dysfunction are not universally present early in disease and frequently progress, which may indicate a lower sensitivity in detecting early stages. Specificity of autonomic symptoms is probably low-for example, constipation prevalence approximates $30 \%$, compared with a PD prevalence of $1-2 \%$. Markers which are potentially more specific, such as MIBG scintigraphy, are time-consuming and/or expensive.

\section{STAGE 2 MARKERS Depression}

Depression is common in PD and is often found early in the disease course. ${ }^{25}$ Pathophysiology of depression in PD is complex and may involve dysfunction of numerous structures. ${ }^{32}$ Important brainstem nuclei linked to depression in $\mathrm{PD}$ include the dorsal motor nucleus of the vagus, ${ }^{33}$ serotonin neurons of dorsal raphae, ${ }^{32}{ }^{34}$ substantia nigra ${ }^{33} 34$ and catecholaminergic neurons of locus ceruleus. ${ }^{33}$ Some of these structures are involved in Braak Stage 2. ${ }^{13}$ A retrospective cohort study based upon database diagnoses determined that those with a history of depression had a 2.4-fold increased risk of developing PD. ${ }^{35}$ A second national registry cohort found that those with a diagnosis of depression had a 2.4 to 3.2-fold increased risk of developing PD compared with patients with osteoarthritis and diabetes. ${ }^{35}$ The health professionals cohort study found that those with phobic anxiety were at a 1.5 -fold increased risk of developing PD. ${ }^{35}$ Many case-control studies have also shown an increased PD risk in patients with history of depression. ${ }^{35}$ Finally, patients with depression may have abnormalities on SNpc transcranial ultrasound that are similar to PD (see below). ${ }^{34}$ Eventual use of depression as a disease predictor will likely be limited by low specificity-the large majority of persons with depression will never develop PD.

There have also been suggestions that early-life personality traits may be associated with PD. PD patients are often described as sober, reliable and conservative, and persons with PD have low scores on measures of novelty-seeking. Although these traits are considered lifelong, ${ }^{36}$ personality assessment may be biased by recall (recall of historical personality is affected by the present personality). If these personality changes emerge with time, they may be a marker of preclinical PD. Sensitivity and specificity of personality screens have also not been established.

\section{REM sleep behaviour disorder}

REM sleep behaviour disorder (RBD) is characterised by a loss of the normal atonia of REM sleep-affected patients cry out, kick or thrash in association with dream content. ${ }^{37} \mathrm{RBD}$ occurs in approximately a third of patients with $\mathrm{PD}$, with an additional $20-35 \%$ demonstrating asymptomatic loss of REM atonia. ${ }^{38}$ RBD is common in MSA and LBD, and is seen occasionally in other parkinsonian neurodegenerative diseases. ${ }^{37}$ It has an unexplained striking male predominance. Prevalence has not been directly estimated, but estimations of sleep injury due to RBD range from $0.4 \%$ in persons over $70-0.5 \%$ of the general population. ${ }^{37} \mathrm{RBD}$ has been predominantly linked with lesions in the brainstem, especially pontine areas such as the perilocus ceruleus and putative REM atonia nuclei analogous to the sublateraldorsal nucleus in mice. ${ }^{39}$ These pontine areas correspond most closely to Braak stage 2 .

Several prospective studies have examined the risk of neurodegenerative disease in persons with idiopathic RBD (ie, RBD without evidence of neurodegeneration). ${ }^{40-42}$ The risk of developing a neurodegenerative disease in idiopathic $\mathrm{RBD}$ ranges from 19 to $38 \%$ at 5 years of follow-up, and from 40 to $65 \%$ after 10 years. Approximately half develop PD, and half develop dementia (most, if not all, of these are LBD). This high risk and long latency make RBD ideal markers for predicting PD. The high conversion rate to disease suggests the need for periodic followup examination, in order to detect treatable manifestations of disease early. In addition, the high conversion rate allows other potential predictors of PD to be studied; studies have demonstrated abnormalities in olfaction, colour vision, autonomic symptoms, MIBG scintigraphy and SNpc function in patients with RBD. ${ }^{31}{ }^{43-45}$ Cognitive abnormalities are also found early in $\mathrm{RBD}$, consistent with its potential as a predictor of dementia. ${ }^{46} \mathrm{It}$ is unclear whether patients who are abnormal on these markers are at a higher risk of $\mathrm{PD}$, but ongoing prospective studies should provide early answers in the next few years. A recent study has suggested that degree of sleep atonia loss, a polysomnographic marker of RBD severity, may predict a higher risk of Parkinson's disease in patients with idiopathic RBD. ${ }^{76}$

However, there are again caveats. First, not every PD patient has RBD; RBD may be associated with a specific PD subtype characterised by male sex, akinetic-rigid disease subtype, less medication response, more autonomic manifestations, EEC slowing and impaired cognitive function. ${ }^{47-50}$ If this indicates a different underlying pathophysiological process, findings in RBD patients may apply less well to those who develop PD without RBD. Second, in an age of neuroprotection, RBD will need to be screened for on a large scale. Two screening questionnaires for RBD have been designed. The first, a 10-item 
questionnaire, reported a sensitivity of $96 \%$ and specificity of $92 \%$ in normal controls. ${ }^{51}$ However, in patients with sleep complaints, specificity was only $56 \%$, with misdiagnosis mainly due to behaviours such as talking in one's sleep. A Japanese version of the same questionnaire found excellent sensitivity and specificity compared with controls (89\% and 97\%) and compared with patients with obstructive sleep apnoea (89\% and $91 \%)^{52}$ A second 13 -item questionnaire recently obtained a sensitivity of $82 \%$ with a specificity of $87 \%{ }^{53}$ for RBD. A subset of seven of these questions directed specifically at dreamenactment behaviour showed similar sensitivity and specificity (88\% and $81 \%$ - -this shortened questionnaire may be useful in large-scale cohort studies. Note that in a general population screening programme, a specificity even as good as $90 \%$ may be insufficient, as performing confirmatory polysomnogram in $10 \%$ of the population would be very labour-intensive and expensive. Finally, whether isolated REM sleep atonia without behavioural manifestations predicts PD is unknown-since these patients do not have symptoms, it is impossible to screen their RBD using questionnaires.

Despite these caveats, $\mathrm{RBD}$ has considerable potential as a marker. The high conversion rate to disease implies a marker with immediate clinical application; if a safe and effective neuroprotective agent were developed tomorrow, RBD patients may have to consider taking it. Also, patients with RBD may be the ideal candidates for clinical trials of neuroprotective agents, since their earlier stage of neurodegeneration provides an additional window of opportunity.

\section{Other sleep disorders}

Patients with PD have many other sleep manifestations, including insomnia and excessive daytime somnolence. ${ }^{25}$ These abnormalities are due to degeneration in diffuse structures, which include pontine structures that degenerate in Stage 2 disease. ${ }^{5}$ In the Honolulu-Asia ageing study, a single question assessing excessive daytime sleepiness was asked. ${ }^{54}$ Of those who reported sleepiness, the OR of PD was 2.8. However, only 235 of the 244 patients who reported sleepiness developed PD, so the utility of this question as a screen for PD is unclear. There have been no prospective studies assessing insomnia as a marker for PD.

\section{STAGE 3 MARKERS}

\section{Dopaminergic PET and SPECT imaging}

Dopaminergic innervation from the SNpc can be measured using radiolabelled ligands that label either pre- or postsynaptic dopaminergic terminals. ${ }^{55}$ Dopaminergic PET and SPECT have a very high sensitivity and specificity for parkinsonism (however, distinguishing PD from other causes of parkinsonism is limited). ${ }^{55}$ The reliability of dopaminergic imaging is such that most patients clinically diagnosed as having PD who have normal scans (ie, 'SWEDD's') probably do not have PD. ${ }^{56}$ By extrapolating backwards from patients with early PD, studies have estimated that abnormalities may be measurable approximately 4-7 years before clinical symptoms. ${ }^{4575}$ Heterozygotes for parkin and PINK1 mutations may have abnormalities of Fluoro-dopa PET and raclopride binding ${ }^{59}$ (although whether parkin and PINK1 heterozygosity increase PD risk is unclear). Recent studies have documented loss of dopaminergic function on PET scanning in asymptomatic carriers of LRRK2 mutations, which in one case progressed with development of clinical PD. ${ }^{60}$ Patients with idiopathic RBD also can have abnormalities on dopaminergic imaging (although these are found only in a minority of cases). ${ }^{44}$ As mentioned above, hyposmic relatives of PD patients show decreased dopaminergic innervation. ${ }^{19}$ Dopaminergic innervation directly measures SNpc function, and therefore should identify patients whose progression does not follow the Braak model. However, despite compelling reasons to suggest predictive potential, no prospective studies have yet directly assessed predictive value of dopaminergic imaging in preclinical sporadic PD.

There are some important barriers to eventual application of PET and SPECT in an age of neuroprotection. First, procedures are expensive (and involve injection of 'radioactive' substances that some patients might refuse). This restricts their use to highrisk groups, or for use as a secondary screen. Consistent with their status as a stage 3 marker, there may be limited lead time (ie, 4-7 years). Diagnostic accuracy in premotor stages is also not established-although dopaminergic imaging is well established in clinically established PD, reliably defining subtle preclinical abnormalities may be less reliable.

\section{Transcranial ultrasound}

Transcranial ultrasound (TCS) imaging of the substantia nigra has promise as a non-invasive and inexpensive neuroimaging predictive marker. Approximately $80-90 \%$ of $\mathrm{PD}$ patients have abnormal hyperechogenicity of the SN. ${ }^{61}$ Hyperechogenicity is found early in PD and may help in differential diagnosis of equivocal parkinsonian signs. ${ }^{61}$ TCS is normal in MSA and PSP. Of special interest, one study found that $60 \%$ of clinically normal persons with hyperechogenicity had 18F-dopa uptake below normal, ${ }^{62}$ and hyperechogenicity has been correlated with mild motor slowing in elderly persons free of $\mathrm{PD},{ }^{63}$ suggesting that there are abnormalities in possible preclinical PD. Up to $40 \%$ of patients with idiopathic RBD have abnormalities on $\mathrm{TCS}^{64}$ (this number, although clearly higher than controls, is not near $100 \%$, perhaps suggesting that not all idiopathic RBD patients are destined to develop PD). In addition, controls with abnormal TCS have olfactory dysfunction and depression. ${ }^{65}$

There is some evidence that hyperechogenicity, rather than being a true marker of preclinical $\mathrm{PD}$, may indicate a lifelong risk state for PD. First, abnormalities can be detected in $9 \%$ of young healthy adults ${ }^{62}$ (however, note there is no direct evidence that young adults with abnormal TCS at increased risk-PD patients could develop TCS abnormalities de novo). Second, there is no correlation between the degree of hyperechogenicity and degree of dopaminergic innervation ${ }^{66}$; nor does hyperechogenicity progress as disease progresses, ${ }^{67}$ which would be expected if TCS was a direct marker of neurodegeneration.

TCS has the advantages of non-invasiveness and relatively low cost. On the other hand, it is technician-dependent, and not all patients have adequate bone windows to allow imaging. So far, it has not been assessed outside research laboratories. Nonetheless, its high sensitivity in PD is promising.

\section{Quantitative motor measures}

At present, the diagnosis of parkinsonism is clinical and based upon subjective evaluation. This has led several investigators to try using quantitative motor measures to detect subtle motor changes. Potential quantitative motor measures include the alternate tap test, Purdue PegBoard, precision grip and lift task, alternate finger tap, and Timed up and Go. ${ }^{43}$ Most quantitative motor measures have not been studied for identification of preclinical disease. One study looked at wrist movements in patients with equivocal signs of parkinsonism, in a battery that included measurement of olfaction and mood. ${ }^{68}$ The battery 
identified eventual PD diagnosis with $92 \%$ sensitivity and $68 \%$ specificity. However, as the battery was applied in persons who already had possible parkinsonism, it did not test a true preclinical state. Quantitative measures are also abnormal in idiopathic RBD, although less dramatically than non-motor markers. ${ }^{43}{ }^{45}$ A potential limitation to quantitative motor testing is that subtle motor slowing occurs in up to $40 \%$ of elderly persons, ${ }^{69}$ suggesting suboptimal specificity. It is unclear whether these measures will perform better than clinical examination in distinguishing incidental mild parkinsonian signs from clinical disease. Finally, as with other stage 3 markers, it is unclear how much lead time would be gained.

\section{Other potential predictors}

Numerous visual changes occur in PD, many early in the course of the disease. Loss of colour vision is found early in $\mathrm{PD}$ and may be due to retinal degeneration. ${ }^{70}$ However, reliability of colour vision loss in early PD is unclear, especially since abnormalities progress with time. ${ }^{71}$ Contrast sensitivity loss is also found early in $\mathrm{PD} .{ }^{70}$ No visual tests have been performed in persons before and after development of $\mathrm{PD}$, but findings of abnormal colour vision in idiopathic $\mathrm{RBD}^{43}$ suggest that visual changes may have potential as a predictor.

Other studies in idiopathic RBD suggest additional potential predictors of PD. Patients with idiopathic RBD have increased $\theta$ power and generalised slowing on EEG. ${ }^{72}$ This finding is similar to what is seen in dementia, suggesting that it is more likely to be a marker of preclinical dementia than PD. Patients with idiopathic RBD also have abnormalities on cognitive testing-these are similar to those seen in PD dementia/LBD, again consistent with a marker of preclinical dementia. ${ }^{73}$ Subtle frontal executive changes are also often found in early $\mathrm{PD}$, are often reversible with levodopa therapy and may have a different pathophysiological basis from that of PD dementia-these changes are relatively subtle, with overlap between patients and controls, suggesting insufficient sensitivity and specificity for detection of PD. Finally, there may be a PD-specific network of changes of whole-glucose utilisation on PET imaging. ${ }^{74}$ Similar changes were found in a group of idiopathic RBD patients, suggesting that these findings may be present in preclinical disease. ${ }^{75}$ Further follow-up of patients with RBD may be able to determine whether any of these factors can predict disease.

\section{CONCLUSION}

Although there is considerable promise for clinical predictors of $\mathrm{PD}$, no single marker is presently able to predict the disease with good reliability and sensitivity. In planning for the eventual use of predictive markers, it may become necessary to design both simple non-invasive screens which can be applied to the general population, and more specific confirmatory tests for those who screen positive. Therefore, multiple lines of investigation should be encouraged. The instant that effective neuroprotective therapy becomes available, detection of early stages of disease will become critically important-therefore, the time to develop reliable predictive methods is now.

Funding Fonds de la recherche en santé du Québec 500, rue Sherbrooke Ouest, Bureau 800 Montréal (Québec) Canada H3A 3C6.

Competing interests $\mathrm{JM}$ received personal compensation as consultant (Boehringer Ingelheim, Servier, Shire Biochem), speaker (Boehringer, Shire), and received financial support for research activities from Sanofi Synthelabo, GlaxoSmithKline. RBP received personal compensation as a consultant and speaker for Teva Neuroscience.

Provenance and peer review Not commissioned; externally peer reviewed.

\section{REFERENCES}

1. Tibben A. Predictive testing for Huntington's disease. Brain Res Bull 2007; 72:165-71

2. de Lau LM, Breteler MM. Epidemiology of Parkinson's disease. Lancet Neurol 2006;5:525-35.

3. Tanner CM, Ottman R, Goldman SM, et al. Parkinson disease in twins: an etiologic study. JAMA 1999;281:341-6.

4. Morrish PK, Rakshi JS, Bailey DL, et al. Measuring the rate of progression and estimating the preclinical period of Parkinson's disease with [18F]dopa PET. J Neurol Neurosurg Psychiatry 1998;64:314-19.

5. Braak H, Del Tredici K, Rub U, et al. Staging of brain pathology related to sporadic Parkinson's disease. Neurobiol Aging 2003;24:197-211.

6. Braak H, Sastre M, Bohl JR, et al. Parkinson's disease: lesions in dorsal horn layer I, involvement of parasympathetic and sympathetic pre- and postganglionic neurons. Acta Neuropathol 2007;113:421-9.

7. Zaccai J, Brayne C, McKeith I, et al. Patterns and stages of alpha-synucleinopathy: Relevance in a population-based cohort. Neurology 2008;70:1042-8.

8. Halliday G, Hely M, Reid W, et al. The progression of pathology in longitudinally followed patients with Parkinson's disease. Acta Neuropathol 2008:115:409-15.

9. Dickson DW, Fujishiro $H$, Delledonne A, et al. Evidence that incidental Lewy body disease is pre-symptomatic Parkinson's disease. Acta Neuropathol 2008;115:437-44.

10. Kalaitzakis ME, Graeber MB, Gentleman SM, et al. The dorsal motor nucleus of the vagus is not an obligatory trigger site of Parkinson's disease: a critical analysis of alpha-synuclein staging. Neuropathol App/ Neurobiol 2008;34:284-95.

11. Parkkinen L, Pirttila T, Alafuzoff I. Applicability of current staging/categorization of alpha-synuclein pathology and their clinical relevance. Acta Neuropathol 2008;115:399-407.

12. Zhang J, Sokal I, Peskind ER, et al. CSF multianalyte profile distinguishes Alzheime and Parkinson diseases. Am J Clin.Pathol 2008:129:526-9.

13. Braak H, Del TK. Invited article: nervous system pathology in sporadic Parkinson disease. Neurology 2008; 70:1916-25.

14. Hawkes C. Olfaction in neurodegenerative disorder. Mov Disord 2003:18:364-72

15. Olichney JM, Murphy C, Hofstetter CR, et al. Anosmia is very common in the Lewy body variant of Alzheimer's disease. J Neurol Neurosurg Psychiatry 2005:76:1342-7.

16. Doty RL, Shaman P, Dann M. Development of the University of Pennsylvania smell identification test: a standardized microencapsulated test of olfactory function. Physiol Behav 1984;32:489-502.

17. Hummel T, Sekinger B, Wolf SR, et al. 'Sniffin' Sticks': olfactory performance assessed by the combined testing of odour identification, odor discrimination and olfactory threshold. Chemical Senses 1997;22:39-52.

18. Berendse HW, Booij J, Francot CM, et al. Subclinical dopaminergic dysfunction in asymptomatic Parkinson's disease patients' relatives with a decreased sense of smell. Ann Neurol 2001;50:34-41.

19. Ponsen MM, Stoffers D, Booij J, et al. Idiopathic hyposmia as a preclinical sign of Parkinson's disease. Ann Neurol 2004:56:173-81.

20. Ponsen MM, Stoffers D, Twisk JW, et al. Hyposmia and executive dysfunction as predictors of future Parkinson's disease: a prospective study. Mov Disord 2009;24:1060-5

21. Haehner A, Hummel T, Hummel C, et al. Olfactory loss may be a first sign of idiopathic Parkinson's disease. Mov Disord 2007:22:839-42.

22. Ross GW, Petrovitch $\mathrm{H}$, Abbott RD, et al. Association of olfactory dysfunction with risk for future Parkinson's disease. Ann Neurol 2008;63:167-73.

23. Marras C, Goldman S, Smith A, et al. Smell identification ability in twin pairs discordant for Parkinson's disease. Mov Disord 2005;20:687-93.

24. Orimo S, Takahashi A, Uchihara T, et al. Degeneration of cardiac sympathetic nerve begins in the early disease process of Parkinson's disease. Brain Pathol 2007:17:24-30.

25. Chaudhuri KR, Healy DG, Schapira AH. Non-motor symptoms of Parkinson's disease: diagnosis and management. Lancet Neurol 2006;5:235-45.

26. Abbott RD, Petrovitch $\mathrm{H}$, White LR, et al. Frequency of bowel movements and the future risk of Parkinson's disease. Neurology 2001:57:456-62.

27. Savica R, Carlin JM, Grossardt BR, et al. Medical records documentation of constipation preceding Parkinson disease: a case-control study. Neurology 2009:73:1752-8

28. Lanfranchi PA, Fradette L, Gagnon JF, et al. Cardiac autonomic regulation during sleep in idiopathic REM sleep behavior disorder. Sleep 2007;30:1019-25.

29. Ferini-Strambi L, Oldani A, Zucconi M, et al. Cardiac autonomic activity during wakefulness and sleep in REM sleep behavior disorder. Sleep 1996;19:367-9.

30. Spiegel J, Hellwig D, Farmakis G, et al. Myocardial sympathetic degeneration correlates with clinical phenotype of Parkinson's disease. Mov Disord 2007:22:1004-8

31. Miyamoto T, Miyamoto M, Inoue Y, et al. Reduced cardiac 123I-MIBG scintigraphy in idiopathic REM sleep behavior disorder. Neurology 2006;67:2236-8.

32. Lieberman A. Depression in Parkinson's disease-a review. Acta Neurol Scand 2006:113:1-8.

33. Frisina PG, Haroutunian V, Libow LS. The neuropathological basis for depression in Parkinson's disease. Parkinsonism Relat Disord 2009;15:144-8. Epub 2008 Jun 20.

34. Walter U, Hoeppner J, Prudente-Morrissey L, et al. Parkinson's disease-like midbrain sonography abnormalities are frequent in depressive disorders. Brain 2007:130:1799-807. 
35. Ishihara L, Brayne C. A systematic review of depression and mental illness preceding Parkinson's disease. Acta Neurol Scand 2006;113:211-20.

36. Ishihara L, Brayne $\mathrm{C}$. What is the evidence for a premorbid parkinsonian personality: a systematic review. Mov Disord 2006;21:1066-72.

37. Gagnon JF, Postuma RB, Mazza S, et al. Rapid-eye-movement sleep behaviour disorder and neurodegenerative diseases. Lancet Neurol 2006;5:424-32.

38. Gagnon JF, Bedard MA, Fantini ML, et al. REM sleep behavior disorder and REM sleep without atonia in Parkinson's disease. Neurology 2002;59:585-9.

39. Boeve BF, Silber MH, Saper CB, et al. Pathophysiology of REM sleep behaviour disorder and relevance to neurodegenerative disease. Brain 2007; 130:2770-88.

40. Iranzo A, Molinuevo JL, Santamaria J, et al. Rapid-eye-movement sleep behaviour disorder as an early marker for a neurodegenerative disorder: a descriptive study. Lancet Neurol 2006;5:572-7.

41. Schenck CH, Bundlie SR, Mahowald MW. Delayed emergence of a parkinsonian disorder in $38 \%$ of 29 older men initially diagnosed with idiopathic rapid eye movement sleep behaviour disorder. Neurology 1996:46:388-93.

42. Postuma RB, Gagnon JF, Vendette $M$, et al. Quantifying the risk of Neurodegenerative Disease in Idiopathic REM sleep behavior disorder. Neurology 2009:72:1296-300.

43. Postuma RB, Lang AE, Massicotte-Marquez J, et al. Potential early markers of Parkinson disease in idiopathic REM sleep behavior disorder. Neurology 2006;66:845-51.

44. Stiasny-Kolster K, Doerr Y, Moller JC, et al. Combination of 'idiopathic' REM sleep behaviour disorder and olfactory dysfunction as possible indicator for alphasynucleinopathy demonstrated by dopamine transporter FP-CIT-SPECT. Brain 2005;128:126-37.

45. Postuma RB, Gagnon JF, Vendette $\mathrm{M}$, et al. Markers of neurodegeneration in idiopathic REM sleep behavior disorder and Parkinson disease. Brain 2009;132 (Pt 12):3298-307.

46. Massicotte-Marquez J, Decary A, Gagnon JF, et al. Executive dysfunction and memory impairment in idiopathic REM sleep behavior disorder. Neurology 2008;70:1250-7.

47. Postuma RB, Gagnon JF, Vendette M, et al. Manifestations of Parkinson disease differ in association with REM sleep behavior disorder. Movement Disorders 2008;23:1665-72.

48. Postuma RB, Gagnon JF, Vendette M, et al. REM sleep behavior disorder in Parkinson's disease is associated with specific motor features. J Neurol Neurosurg Psychiatry 2008;79:1117-21.

49. Vendette M, Gagnon JF, Decary A, et al. REM sleep behavior disorder predicts cognitive impairment in Parkinson's disease without dementia. Neurology 2007:69:1843-9.

50. Kumru H, Santamaria J, Tolosa E, et al. Relation between subtype of Parkinson's disease and REM sleep behavior disorder. Sleep Med 2007;8:779-83.

51. Stiasny-Kolster K, Mayer G, Schafer S, et al. The REM sleep behavior disorder screening questionnaire-a new diagnostic instrument. Mov Disord 2007;22:2386-93.

52. Miyamoto T, Miyamoto M, Iwanami M, et al. The REM sleep behavior disorder screening questionnaire: validation study of a Japanese version. Sleep Med 2009;10:1151-4.

53. Li SX, Wing YK, Lam SP, et al. Validation of a new REM sleep behavior disorder questionnaire (RBDQ-HK). Sleep Med 2010;11:43-8.

54. Abbott RD, Ross GW, White LR, et al. Excessive daytime sleepiness and subsequent development of Parkinson disease. Neurology 2005;65:1442-6.
55. Ravina B, Eidelberg D, Ahlskog JE, et al. The role of radiotracer imaging in Parkinson disease. Neurology 2005;64:208-15.

56. Marek K, Jennings D, Seibyl J. Long-term follow-up of patients with scans without evidence of dopaminergic deficit (SWEDD) in the Elldopa study. Neurology 2005:64 A274. Ref Type: Abstract.

57. Hilker R, Schweitzer K, Coburger S, et al. Nonlinear progression of Parkinson disease as determined by serial positron emission tomographic imaging of striatal fluorodopa F 18 activity. Arch Neurol 2005;62:378-82.

58. Vingerhoets FJ, Snow BJ, Lee CS, et al. Longitudinal fluorodopa positron emission tomographic studies of the evolution of idiopathic parkinsonism. Ann.Neurol 1994;36:759-64.

59. Stoessl AJ. Positron emission tomography in premotor Parkinson's disease. Parkinsonism Relat Disord 2007;13(Suppl 3):S421-4.

60. Nandhagopal R, McKeown MJ, Stoessl AJ. Functional imaging in Parkinson disease. Neurology 2008:70:1478-88.

61. Gaenslen A, Unmuth B, Godau J, et al. The specificity and sensitivity of transcranial ultrasound in the differential diagnosis of Parkinson's disease: a prospective blinded study. Lancet Neurol 2008;7:417-24.

62. Berg D, Becker G, Zeiler B, et al. Vulnerability of the nigrostriatal system as detected by transcranial ultrasound. Neurology 1999:53:1026-31.

63. Berg D, Siefker C, Ruprecht-Dorfler P, et al. Relationship of substantia nigra echogenicity and motor function in elderly subjects. Neurology 2001:56:13-7.

64. Stockner H, Iranzo A, Seppi K, et al. Midbrain hyperechogenicity in idiopathic REM sleep behavior disorder. Movement Disorders 2009;24:1906-9.

65. Berg D, Godau J, Walter U. Transcranial sonography in movement disorders. Lancet Neurol 2008:7:1044-55.

66. Spiegel J, Hellwig D, Mollers MO, et al. Transcranial sonography and [123l]FP-CIT SPECT disclose complementary aspects of Parkinson's disease. Brain 2006;129:1188-93

67. Berg D, Merz B, Reiners K, et al. Five-year follow-up study of hyperechogenicity of the substantia nigra in Parkinson's disease. Mov Disord 2005:20:383-5.

68. Montgomery EB Jr, Lyons K, Koller WC. Early detection of probable idiopathic Parkinson's disease: II. A prospective application of a diagnostic test battery. Mov Disord 2000:15:474-8.

69. Bennett DA, Beckett LA, Murray AM, et al. Prevalence of parkinsonian signs and associated mortality in a community population of older people. $N$ Engl J Med 1996;334:71-6.

70. Price MJ, Feldman RG, Adelberg D, et al. Abnormalities in color vision and contrast sensitivity in Parkinson's disease. Neurology 1992:42:887-90.

71. Muller T, Woitalla D, Peters S, et al. Progress of visual dysfunction in Parkinson's disease. Acta Neurol Scand 2002;105:256-60.

72. Fantini ML, Gagnon JF, Petit D, et al. Slowing of electroencephalogram in rapid eye movement sleep behavior disorder. Ann Neurol 2003;53:774-80.

73. Gagnon JF, Vendette M, Postuma RB, et al. Mild cognitive impairment in rapid eye movement sleep behavior disorder and Parkinson's disease. Ann Neurol 2009;66:39-47.

74. Moeller JR, Eidelberg D. Divergent expression of regional metabolic topographies in Parkinson's disease and normal ageing. Brain 1997;120(Pt 12):2197-206.

75. Mazza S, Soucy JP, Gravel P, et al. Assessing whole brain perfusion changes in patients with REM sleep behavior disorder. Neurology 2006;67:1618-22.

76. Postuma RB, Gagnon JF, Rompre S, et al. Severity of REM atonia loss in idiopathic REM sleep behavior disorder predicts Parkinson's disease. Neurology 2010;74:239-44 